\title{
E-mentoring na pandemia da COVID- 19: percepção dos alunos ingressantes de Fonoaudiologia
}

\author{
E-mentoring during COVID-19 pandemic: Speech \\ Therapy students' perception
}

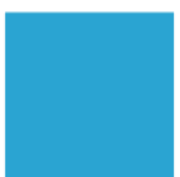

Revista

Extensão em Foco
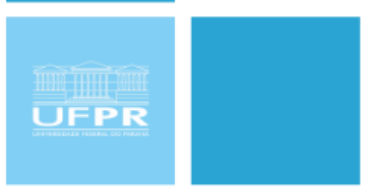

ISSN $2358-7180$

\section{Humberto de Oliveira Simões ${ }^{1}$, Pamela Papile Lunardelo ${ }^{2}$, Victoria Costa Lippi ${ }^{3}$, Aline Epiphanio Wolf ${ }^{4}$}

\section{RESUMO}

O objetivo deste estudo foi avaliar a percepção e benefício dos alunos ingressantes do curso de Fonoaudiologia para o programa e-mentoring, realizado durante o período de isolamento social da pandemia da COVID-19. Foram realizados 15 encontros, durante os meses de março a junho de 2020, de forma online, juntamente com três mentores e uma peer. Após a conclusão do programa, os acadêmicos foram instruídos a responder um formulário sobre a percepção do programa. Como resultados tivemos 26 alunos que responderam ao formulário e todos foram congruentes ao apontarem que o e-mentoring trouxe benefícios ao processo de inserção e adaptação à Universidade. As estratégias de ensino-aprendizado foram facilitadas e os conteúdos discutidos foram adequados, conforme apontamento de $96,2 \%$ dos alunos participantes. Diante da pandemia da COVID-19, 96,2\% também disseram que o programa e-mentoring foi "muito importante" no processo de adaptação ao ambiente universitário. Observa-se que o programa implementado de forma on-line, obteve apontamentos positivos ao ponto de vista dos benefícios trazidos aos alunos no processo de inserção ambiental na Universidade. O e-mentoring além de auxiliar na ambientação e socialização dos alunos com seus pares, favoreceu também estratégias de ensino e aprendizagem.

Palavras-chaves: Educação; Fonoaudiologia; Tutoria; Educação superior; Infecções por Coronavirus.

\section{ABSTRACT}

The aim was to evaluate the perception and benefit of students from the first semester of the Speech Therapy graduation course about the e-mentoring program, carried out during the social isolation of the COVID-19 pandemic. 15 on-line meeting were held, from March to June 2020, together with three mentors and one peer. Upon completion of the program, academics were instructed to answer a form about the perception of the program throughout the semester. The results, 26 students answered the form and all of them agreed that the e-mentoring program brought benefits to the insertion and adaptation process at the University. The teaching and learning strategies were facilitated and the contents discussed were adequate, as indicated by $96.2 \%$ of the students. In the current situation of COVID-19, 96.2\% also said that the e-mentoring program was "very important" in the process of adaptation to the University environment. It is observed that the on-line program implemented, obtained positive notes in terms of the benefits brought to students in the process of University environmental insertion. The e-mentoring not only helped students to get acquainted and socialized with their peers, but also favored teaching and learning strategies.

\footnotetext{
${ }^{1}$ Mestre em ciências. Faculdade de Medicina de Ribeirão Preto (FMRP), Ribeirão Preto, SP, Brasil. E-mail: humbertodeoliveirasimoes@ hotmail.com. Orcid: https://orcid.org/0000-0003-3544-8262.

${ }^{2}$ Mestre em psicobiologia. Faculdade de Filosofia, Ciências e Letras de Ribeirão Preto (FFCLRP), Ribeirão Preto, SP, Brasil. E-mail: paam lunardelo@ hotmail.com. Orcid: https://orcid.org/0000-0003-0382-637X.

${ }^{3}$ Graduação em Fonoaudiologia. Faculdade de Medicina de Ribeirão Preto (FMRP), Ribeirão Preto, SP, Brasil.

E-mail: victorialippi@usp.br. Orcid: https://orcid.org/0000-0002-3228-9596.

${ }^{4}$ Docente e Doutora em ciências. Faculdade de Medicina de Ribeirão Preto (FMRP), Ribeirão Preto, SP, Brasil.

E-mail: alinewolf@fmrp.usp.br. Orcid: https://orcid.org/0000-0001-5006-8482.
} 
Keywords: Education; Speech, Language and Hearing Sciences; Mentoring; Education, Higher; Coronavirus Infections

\section{INTRODUÇÃO}

Ingressar no ambiente universitário pressupõe ao estudante condições de mudanças e adaptações, no que versa ao pessoal, social e no desenvolvimento da identidade autônoma (BELLODI, MARTINS, 2005; REASON, TERENZINI, DOMINGO, 2006). Este processo é amplamente discutido e há o consenso quanto a necessidade de estratégias para facilitar os processos de transição que são inerentes a Universidade. Entretanto, recentemente, a Pandemia do COVID-19 implicou na suspensão das aulas presenciais, alterando as formas de interação e socialização (ELMER, MEPHAM, STADTFELD, 2020).

O princípio de educação integral durante a trajetória acadêmica é de responsabilidade dos serviços de apoio universitário (PANÚNCIO-PINTO, COLARES, 2015). Historicamente, os programas de mentoring foram inseridos no contexto do ensino em saúde nos anos 90 nos Estados Unidos e na Europa (BELLODI, MERTINS, 2005). Atualmente este é bem difundido e sucedido em todo o mundo (RODRÍGUEZ-PLANAS, 2012), em nível nacional era considerado uma nova modalidade na primeira década dos anos 2000, quando era ofertado por 32 escolas médicas brasileiras (AGUILAR-DA-SILVA et al., 2009).

Por definição, os programas de mentoring são parcerias que visam compartilhar conhecimentos, habilidades, informações e perspectivas. A partir disto o intuito é apoiar, nutrir e inspirar o outro, ajudando o crescimento pessoal e profissional (MILLS, 2008). Este tipo de programa pode ser aplicado em qualquer momento da trajetória acadêmica ${ }^{7}$, faz parte dos programas de tutoria e tem como alicerce três componentes básicos: suporte pessoal, educação permanente e aprimoramento profissional para alcançar o desenvolvimento global do estudante (PANÚNCIO-PINTO, COLARES, 2015).

$\mathrm{Na}$ literatura encontram-se alguns estudos que apontam a contribuição da tutoria mentoring nos cursos de fonoaudiologia. Em uma universidade nacional em que o programa é ofertado desde 2009 os efeitos do mentoring foram satisfatórios, segundo a percepção dos estudantes (ALPES, WOLF, 2018; ALPES, CORSI, WOLF, 2019). Em nível internacional, um estudo evidenciou que o suporte e orientação em tarefas são preditores de satisfação na carreira 
(HARRIS, WINSKOWSKI, ENGDAHL, 2007). Os alunos que participaram deste programa mostraram-se mais comprometidos e satisfeitos com o então atual emprego (ALLEN et al., 2004; HARRIS, WINSKOWSKI, ENGDAHL, 2007). Outro estudo identificou que estudantes inseridos em programas de tutoria mentoring apresentaram melhor desempenho na prática clínica em comparação aos que não estavam inseridos, indicando que quanto maior o apoio oferecido, melhor o desempenho (PAMPLONA et al., 2015).

O isolamento social decorrente da pandemia da COVID-19 influenciou negativamente o bem-estar psicológico e saúde mental dos estudantes. Um estudo realizado por pesquisadores suíços descreveu que estudantes universitários se sentiam mais deprimidos, ansiosos, estressados e solitários em apenas duas semanas de cancelamento das aulas presenciais (ELMER, MEPHAM, STADTFELD, 2020). Em um grupo de 2485 estudantes as taxas de estresse pós-traumático foram de $2,7 \%$ e de $9 \%$ de depressão após um mês de pandemia (TANG et al., 2020). Outro estudo identificou um aumento de 42,5\% de ansiedade comparado ao mesmo período do ano anterior (KAPAROUNAKI et al., 2020). Segundo Elmer e colaboradores (ELMER, MEPHAM, STADTFELD, 2020), estas informações auxiliam na composição de grupos de apoio e programas de mentoring. Ainda, reforçam que os alunos ingressantes não tiveram a oportunidade de desenvolver laços e as iniciativas da Universidade de promover encontros on-line poderiam facilitar este processo.

A pandemia da COVID-19 promoveu a modificação de programas de mentoring presencial para a modalidade on-line. Resultados preliminares indicam que este novo formato pode ser uma alternativa adequada e viável neste momento de isolamento (GERNERT et al., 2020). Kazerooni e colaboradores (2020) ofertaram o e-mentoring a 371 estudantes do primeiro ano do curso de medicina, $71 \%$ destes relataram que o programa promoveu impacto positivo em sua adaptação e na solução de problemas. Ainda, os estudantes declararam que a oferta do mentoring remoto os ajudou a lidar com a ansiedade e estresse promovidos pela pandemia. $\mathrm{O}$ e-mentoring constitui um espaço em que é possível identificar como a pandemia impacta a vida dos estudantes. A partir da oferta do programa on-line, um estudo identificou que $44 \%$ dos universitários apresentaram sintomas leves a graves de depressão, menor motivação para estudar, sentiram-se sobrecarregados e preocupados com a influência negativa deste período em seus estudos. Os autores não estabeleceram relações causais, mas enfatizaram o impacto nocivo no bem-estar dos estudantes, e que estas devem ser questões abordadas pelos mentores (GUSE et al., 2020). A mentoria na modalidade on-line permite a prestação de cuidados e apoio aos estudantes, fundamentais para a promoção de saúde mental destes (GERNERT et al, 2020). 
Gernert et al. (2020) identificaram que a experiência de modificação de três programas de mentoring presencial para a modalidade on-line foi importante para o crescimento profissional dos mentores e para o alívio psicológicos dos alunos que ingressaram na universidade. Os autores verificaram que o número de participantes foi comparável com a modalidade presencial e que houve alta satisfação entre os estudantes com o programa.

Com isto, objetivou-se avaliar a percepção de alunos do primeiro ano do curso de Fonoaudiologia quanto a importância do programa de tutoria mentoring on-line (e-mentoring), oferecido no primeiro semestre de 2020 , durante os quatro primeiros meses de isolamento social em nível nacional. E, assim, verificar se a estratégia de oferta da mentoria de forma remota manteria o benefício semelhante ao modelo presencial.

\section{MÉTODO}

Estudo descritivo-exploratório, com abordagem quali-quantitativa. O presente trabalho foi apresentado ao Comitê de Ética em Pesquisa institucional, na qual obteve dispensa de análise ética em 04 de setembro de 2020.

A oferta do programa de tutoria mentoring é idealizada, organizada e gerida pelo Centro de Apoio Educacional e Psicológico (CAEP) junto a Faculdade de Medicina de Ribeirão Preto, (FMRP) no contexto de assistência ao estudante. No curso de fonoaudiologia uma docente da instituição, dois ex-alunos e atualmente pós-graduandos (mentores) e uma aluna do segundo ano do curso (peer) realizaram a capacitação para o programa mentoring. A partir disto, instituise o grupo de mentoria com os alunos ingressantes do ano de 2020. Os encontros foram realizados no formato on-line de março a julho de 2020.

Para exploração dos dados, foi aplicado um instrumento semiestruturado, elaborado para aplicação com os estudantes do primeiro semestre do curso de Fonoaudiologia (ALPES, CORSI, WOLF, 2019). Os 30 estudantes foram abordados, no último encontro on-line, por meio do questionário elaborado através da plataforma do Google Forms, formado por nove questões fechadas e por uma questão aberta. Foi acrescida uma questão relacionada a COVID19 e o cenário atual de ensino frente a mentoria realizada (Quadro 1). 
Quadro 1 - Questionário aplicado aos alunos elaborado por ALPES, CORSI, WOLF (2019), acrescido de uma questão sobre o cenário atual de ensino na pandemia do COVID-19.

\begin{tabular}{|c|c|c|c|c|}
\hline Questões & \multicolumn{4}{|c|}{ Opções de respostas } \\
\hline $\begin{array}{l}\text { Q1. Trouxe algum tipo de } \\
\text { benefício para você? }\end{array}$ & & Sim & \multicolumn{2}{|c|}{ Não } \\
\hline $\begin{array}{l}\text { Q2. Ajudou no processo de } \\
\text { ingresso e } \\
\text { Universidade? }\end{array}$ & & Sim & \multicolumn{2}{|c|}{ Não } \\
\hline $\begin{array}{l}\text { Q3. Beneficiou a resolução de } \\
\text { questões e/ou problemas } \\
\text { relacionados à universidade? }\end{array}$ & & Sim & \multicolumn{2}{|c|}{ Não } \\
\hline $\begin{array}{l}\text { Q4. Influenciou na manutenção } \\
\text { de estratégias } \\
\text { estudos/aprendizagem } \\
\text { disciplinas? }\end{array}$ & & Sim & \multicolumn{2}{|c|}{ Não } \\
\hline $\begin{array}{l}\text { Q5. Os conteúdos propostos } \\
\text { foram adequados? }\end{array}$ & & Sim & \multicolumn{2}{|c|}{ Não } \\
\hline $\begin{array}{l}\text { Q6. Os profissionais (professores } \\
\text { e/ou colaboradores) eram } \\
\text { preparados? }\end{array}$ & & Sim & \multicolumn{2}{|c|}{ Não } \\
\hline $\begin{array}{l}\text { Q7. Após o término, evidenciou } \\
\text { benefícios a partir da } \\
\text { experiência? }\end{array}$ & & Sim & \multicolumn{2}{|c|}{ Não } \\
\hline $\begin{array}{l}\text { Q8. É um dever da Universidade } \\
\text { oferecer a Mentoria Acadêmica } \\
\text { aos alunos? }\end{array}$ & & $\operatorname{Sim}$ & \multicolumn{2}{|c|}{ Não } \\
\hline $\begin{array}{l}\text { Q9. Diante da nova realidade } \\
\text { (COVID-19), como você } \\
\text { descreveria a importância da } \\
\text { Mentoria Acadêmica no seu } \\
\text { processo de adaptação à } \\
\text { Universidade? }\end{array}$ & $\begin{array}{c}\text { Muito } \\
\text { importante }\end{array}$ & $\begin{array}{l}\text { Razoavelmente } \\
\text { importante }\end{array}$ & $\begin{array}{l}\text { Pouco } \\
\text { importante }\end{array}$ & Indiferente \\
\hline
\end{tabular}

Fonte: autoria própria (2020)

Em termos quantitativos nas questões fechadas dicotômicas (sim/não) ou a de opção, foi utilizada a frequência simples para cada uma das respostas. Para os dados qualitativos, utilizamos a análise de conteúdo das respostas da questão aberta. 


\section{RESULTADOS}

Os encontros on-line foram realizados no primeiro semestre letivo de 2020, com utilização da plataforma do Google Meet. Os encontros foram semanais, com duração aproximada de 60 minutos, em horário exclusivo. No total, foram 15 encontros realizados no período de março a julho, nos quais foram ofertados temas específicos e de livre demanda.

A adesão média foi de 20 estudantes a cada encontro, nos quais eram sempre mantidas temáticas voltados a atuação fonoaudiológica, clínica e acadêmica, além de formação de carreira, dificuldades, dúvidas e curiosidades no que se refere contexto universitário. Os encontros tiveram três direcionamentos que sempre foram linha de direcionamento para os mentores no processo de condução, foram eles: queixas - os alunos aproveitavam o momento para poder buscar compreender as estruturas de aula e atividades que lhes eram passadas ao longo da semana e suas eventuais dificuldades; soluções - nesse momento, os alunos buscavam por estratégias, guiadas pelos mentores e peer, a fim de poder reestruturar a forma de estudar e lidar com as situações opositoras; e, por fim, descontração - os minutos finais eram reservados para tópicos voltados a desconstrução rígida da universidade e suas atividades.

Como parte final da mentoria, obteve-se retorno de resposta do questionário de 26 acadêmicos, cuja súmula das respostas, em porcentagem, encontram-se na Figura 1. A questão aberta foi uma forma de trazer aos alunos a oportunidade de explanar sua opinião frente aos benefícios da mentoria em seu semestre realizado à distancia. As respostas foram diversificadas e abaixo estão três delas:

- "A mentoria foi uma experiência incrivel e inigualável. Me abriu portas pra conhecer a turma, fazer amizades, me sentir acolhida, saber que existe um espaço onde você possa ser aluno e expressar sentimentos mesmo na relação professor-aluno, é muito bom. Em particular foi de extrema importância, já que eu não cheguei a ir até [cidade] e não conheci os professores e a turma toda. Acho que é um espaço muito bem aproveitado e nesse momento atípico o aproveitamento foi grande, viso que todo mundo precisava se ajudar e ao mesmo tempo aprender. Foi ótimo."

- "Num momento tão tenso, longe da minha família e me adaptando ao ensino remoto, a mentoria foi um alívio e muita das vezes, o incentivo pra continuar! Foram momentos maravilhosos de conversa, risadas e resolução de problemas. Gratidão." 
- “A mentoria me ajudou muito, principalmente nesse período de aulas on-line, porque me mostrava a diversidade de caminhos que eu podia seguir para resolver problemas relacionados à faculdade, às disciplinas e também à vida - com mais calma. Além disso, proporcionou momentos inesquecíveis que fizeram com que eu ficasse mais feliz e animada com o curso, por mais difícil que aparentou ser pra mim aprender à distância. [Nome dos mentores] me ajudaram a crescer, foram essenciais para minha vida profissional e pessoal, só tenho a agradecer!”

Figura 1 - Distribuição percentual das respostas do questionário respondidos pelos acadêmicos $(n=26)$

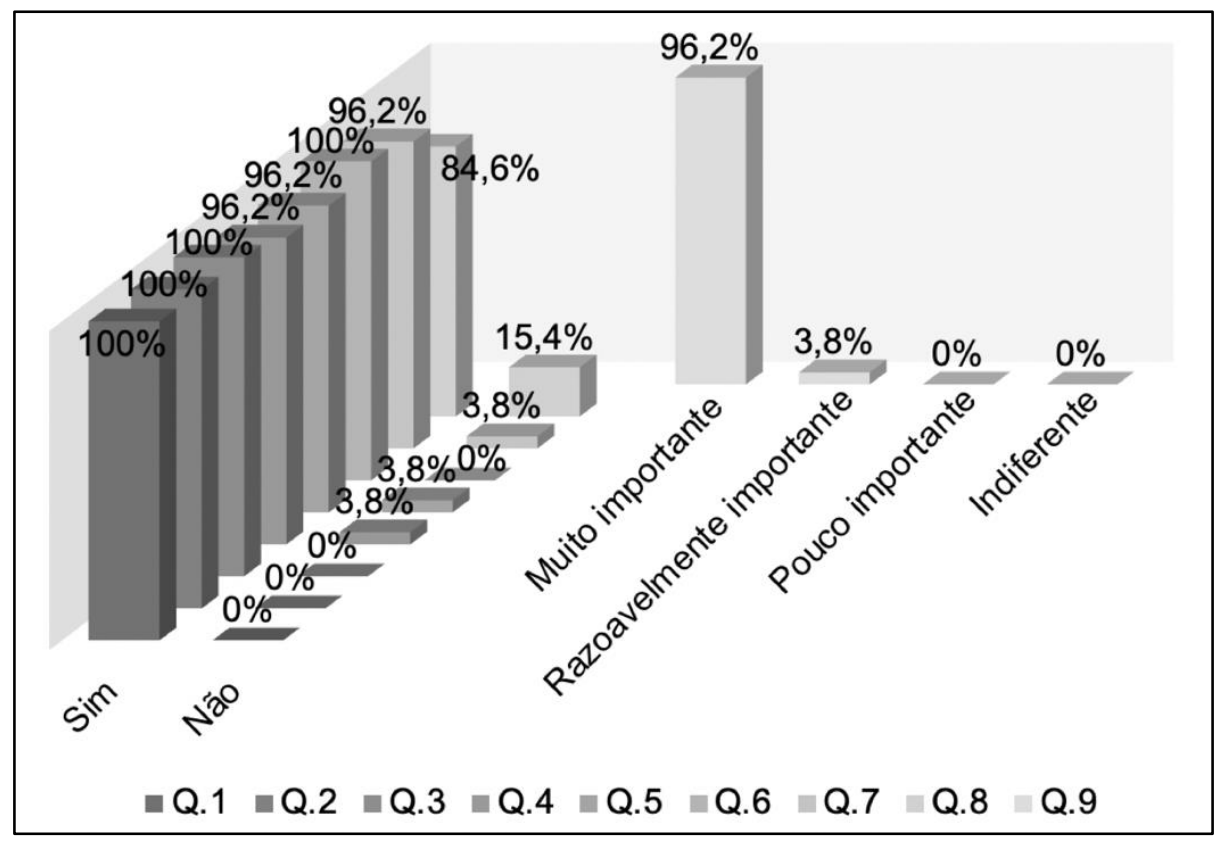

Fonte: autoria própria (2020)

\section{DISCUSSÃO}

Os programas mentoring visam apoiar, nutrir e inspirar jovens estudantes ${ }^{7}$. Buscando alcançar o desenvolvimento global deste por meio do suporte pessoal, educação permanente e aprimoramento profissional (PANÚNCIO-PINTO, COLARES, 2015). 
O programa de e-mentoring retratado no presente trabalho obteve resultados positivos, mostrou benefício aos estudantes tutelados, como declarado por estes. Todos os estudantes concordaram que o programa favoreceu o processo de adaptação a Universidade e 96,2\% acreditam que favoreceu a condução de estratégias para a aprendizagem dos diferentes conteúdos que são oferecidos nas disciplinas que compõem do primeiro semestre do curso de fonoaudiologia. Estes resultados indicam que o programa de e-mentoring cumpriu o objetivo conferido, que profissionais mais experientes forneçam orientação e aconselhamento para “jovens praticantes” (RODRÍGUEZ-PLANAS, 2012).

Os conteúdos oferecidos durante os encontros foram específicos e de livre demanda ao que versa a formação e carreira fonoaudiológica e ao ambiente universitário, de acordo com a maior parte dos estudantes tutelados os conteúdos foram adequados. Quanto aos mentores e peer, $100 \%$ dos estudantes assinalaram que estes estavam preparados para conduzir o programa mentoring. Uma vez que foi apontado pelos calouros que os envolvidos na tutoria estavam preparados para atividade, podemos então entender que a capacitação previamente oferecida foi fundamental para que os encontros fossem conduzidos de acordo com o que visa o programa, de modo que realmente auxiliasse os estudantes. Com isto, reforçasse a necessidade de serviços de apoio universitário qualificados e estruturados com diferentes profissionais, como psicólogos e pedagogos, que possam orientar os mentores (PANÚNCIO-PINTO, COLARES, 2015).

Embora bem estabelecido que é de responsabilidade dos serviços de apoio universitário garantir a educação integral durante a trajetória acadêmica (PANÚNCIO-PINTO, COLARES, 2015), 15,4\% dos estudantes declaram acreditar não é dever da Universidade oferecer a mentoria acadêmica aos alunos. É possível que pelo ingresso recente ainda não tenham compreendido que é um direito fundamental ter vias de acesso a serviços que acolham as demandas especificas referentes a cada período de formação (PANÚNCIO-PINTO, COLARES, 2015). Embora 84,6\% dos estudantes tenham assinalado como positivo para esta questão, é necessário que o tema seja retomado em outros encontros do e-mentoring e que seus objetivos sejam melhores pontuados.

A nova realidade decorrente da Pandemia da COVID-19 promoveu altos índices de sentimentos, emoções e pensamentos negativos após o início do isolamento social e suspensão das aulas presenciais (ELMER, MEPHAM, STADTFELD, 2020; TANG et al., 2020; KAPAROUNAKI et al., 2020). Em nosso trabalho, a maior parte dos estudantes declarou que programa de mentoria acadêmica foi "muito importante" neste período incomum que 
vivenciamos. Estes resultados são semelhantes aos encontrados por Gernert et al (2020), em que os estudantes se declararam altamente satisfeitos com a prestação de cuidado e apoio ofertados pelo e-mentoring neste momento atípico. Segundo Elmer, Mepham e Stadtfeld (2020), os estudantes ingressantes não tiveram a oportunidade de estabelecer vínculos sociais, sendo ideal que a Universidade promova encontros virtuais, para que isto ocorra. O programa de mentoring em formato remoto, e-mentoring, proporcionou aos ingressantes de fonoaudiologia estes encontros virtuais. Neste cenário os estudantes relacionaram-se com seus próprios pares de turma, favorecendo a convivência presencial no futuro. Além disto, as angústias e dúvidas quanto a este período puderam ser compartilhadas e, em casos em que fosse identificado questões pertinentes a intervenções específicas, pedagógicas ou psicológicas, encaminhadas para profissionais qualificados.

Há uma relação positiva entre a inserção de estudantes de fonoaudiologia em programas mentoring e o desempenho na prática clínica e satisfação na carreira (ALLEN et al., 2004; HARRIS, WINSKOWSKI, ENGDAHL, 2007; PAMPLONA et al., 2015). Em nosso estudo, acreditamos que programa e-mentoring contribuiu para que os estudantes continuassem motivados e atraídos pelo ingresso a universidade e construção de carreira, como pode ser observado nos relatos descritivos. Além disto, acredita-se que o programa mentoring ofertado durante o período de isolamento social irá contribuir de forma exponencial para o melhor desempenho e adaptação destes na retomada das atividades acadêmicas presenciais.

É imprescindível que alguns aspectos sejam reforçados. O primeiro refere-se aos estudantes serem os protagonistas deste processo. Para que haja sucesso no programa de mentoring é necessário que estes participem de forma ativa, comprometida e que estejam abertos a receber ajuda e orientação. Que os mentores tenham conhecimento, respeito, paciência e entusiasmo com o processo. As características de ambos permitem que se estabeleça um vínculo de confiança entre mentores, peer e estudantes (KANASKIE, 2006). O segundo aspecto diz respeito a Universidade ter suporte e preparo adequado para o desenvolvimento do programa, contando com outros profissionais capacitados. E o terceiro, versa quanto a participação e disponibilidade da coordenação do curso neste processo, contribuindo nas demandas dos estudantes e orientações, em especial, no atual contexto em que as informações acerca dos próximos passos se alteram constantemente.

Devido a pandemia da COVID-19 foi necessária a modificação do programa de mentoring presencial para a modalidade remota, os resultados deste estudo contribuem para o entendimento quanto a importância desta manutenção. Embora o e-mentoring exclusivo não 
seja consenso na literatura, nossos achados e de estudos anteriores (GERNERT et al., 2020) evidenciam que esta é uma alternativa adequada para o que se propõe. Diante disto, deve-se considerar a permanência de parte do programa em formato remoto, por promover facilidades de interação entre os que dele participam.

Independente do formato de condução, presencial ou via métodos on-line, a distância, os benefícios se tangenciam pelos apontamentos dos estudantes que o realizaram de forma presencial (ALPES, WOLF, 2018; ALPES, CORSI, WOLF, 2019) e os nossos resultados. A eficácia do mentoring é refletida em melhores práticas sociais, acadêmicas e comportamentais para aqueles que dele participam (RODRÍGUEZ-PLANAS, 2012). Com isto, buscou-se assegurar desde o início uma formação integral dos futuros profissionais fonoaudiólogos.

Apesar da facilidade do uso de plataformas on-line, os formulários foram respondidos por 27 alunos dos 30 que foram alvo do programa de mentoria. Essa limitação pode ter acontecido pelo fato do período que estes acadêmicos entraram em férias da universidade. Uma proposta para controle dessa variável, poderia ter sido a implementação de um tempo, dentro do último encontro, para o preenchimento do formulário. Ademais, a constituição amostral foi realizada apenas com acadêmicos do curso de fonoaudiologia, uma vez que este monitoramento final do programa poderia ter sido expandido aos demais cursos da instituição. A adesão dos alunos foi representativa, mas vale considerar que alguns problemas de conexão foram esperados e discretos, comparados a presença dos mesmos.

\section{CONCLUSÃO}

A partir das respostas do questionário aplicado com os acadêmicos ingressantes, podemos concluir que o programa e-mentoring beneficiou o processo de adaptação à Universidade e favoreceu as estratégias de aprendizado destes. Ademais, frente a pandemia da COVID-19, este programa foi apontado como "muito importante" para o auxílio e acompanhamento dos alunos à aclimação dentro do contexto universitário.

\section{AGRADECIMENTOS}


Agradecemos ao programa de mentoria acadêmica da FMRP realizado pelo CAEP em execução através de seus membros, representados de forma mais direta pela psicóloga Gisele Curi de Barros.

\section{REFERÊNCIAS}

AGUILAR-DA-SILVA, R. H.; PERIM, G. L.; ABDALLA, I. G.; COSTA, N. M. D. S. C.; LAMPERT, J. B.; STELLA, R. C. R. Abordagens pedagógicas e tendências de mudanças nas escolas médicas. Revista Brasileira de Educação Médica, v. 33, supl. 1, p. 53-62, 2009.

ALLEN, T.D.; EBY, L. T.; POTEET, M. L.; LENTZ, E.; LIMA, L. Career benefits associated with mentoring for protégeé: a meta-analysis. Journal of Applied Psychology, v. 89 , n. 1, p. 127-36, 2004.

ALPES, M. F.; CORSI, L.; WOLF, A. E. Programa de tutoria ("mentoring"): percepções de graduandos do curso de fonoaudiologia. Revista Extensão em Foco [Internet], n. 19, p. 61-71, 2019.

ALPES, M. F.; WOLF, A. E. Tutoria acadêmica (“mentoring”): relato de experiência de um tutorado a tutor. Revista Extensão em Foco [Internet], n. 16, p. 90-8, 2018.

BELLODI, P. L.; MARTINS, M. A. Tutoria: mentoring na formação médica. São Paulo: Casa do Psicólogo, 2005.

ELMER, T.; MEPHAM, K.; STADTFELD, C. Students under lockdown: Comparisons of students' social networks and mental health before and during the COVID-19 crisis in Switzerland. PLoS One, v. 15, n. 7, p. e0236337, 2020.

GERNERT, J.A.; ZIBOLD, J.; REIK, J.U.; GRAUPE T.; DIMITRIADIS, K. Restructuring career counselling ventures of a mentoring program for medical students in the course of the COVID-19 pandemic. GMS J Med Educ, v.37, Doc.73, 2020.

GUSE, J.; HEINEN, I.; KURRE, J.; MORH, S.; BERGELT, C. Perception of the study situation and mental burden during the COVID-19 pandemic among undergraduate medical students with and without mentoring. GMS J Med Educ, v.37, Doc.72, 2020. 
HARRIS, J. I.; WINSKOWSKI, A. M.; ENGDAHL, B. E. Types of workplace social support in the prediction of job satisfaction. The career development quarterly, v. 56, n. 2, p. 150-6, 2007.

KANASKIE, M, L. Mentoring--a staff retention tool. Critical care nursing quarterly, v. 29, n. 3, p. 248-52, 2006.

KAPAROUNAKI, C. K.; PATSALI, M. E.; MOUSA, D. V.; PAPADOPOULOU, E. V. K.; PAPADOPOULOU, K. K. K.; FOUNTOULAKIS, K. N. University students' mental health amidst the COVID-19 quarantine in Greece. Psychiatry Research, v. 290, p. 113111, 2020.

KAZEROONI, A.R.; AMINI, M.; TABARI, P.; MOOSAVI, M. Peer mentoring for medical students during the COVID-19 pandemic via a social media platform. Med Educ, v. 54, p.762-63, 2020.

MILLS, K. Benefits and characteristics of mentoring students and young professionals. Perspectives on Administration and Supervision, v. 18, n. 2, p. 67-73, 2008.

PAMPLONA, M. C.; YSUNZA, P. A.; SARRE, P.; MORALES, S.; STERLING, M. The effectiveness of mentoring speech and language pathology students when they face patients with cleft palate. International Journal of Pediatric Otorhinolaryngology, v. 79, n. 10, p. 1708-13, 2015.

PANÚNCIO-PINTO, M.; COLARES, M. F. O estudante universitário: os desafios de uma educação integral. Medicina (Ribeirao Preto Online) [Internet], v. 48, n. 3, p. 273-81, 2015.

REASON, R. D.; TERENZINI, P. T.; DOMINGO, R. J. First things first: developing academic competence in the first year of college. Research in Higher Education, v. 47, n. 2, p. 149-75, 2006.

RODRÍGUEZ-PLANAS, N. Longer-Term Impacts of Mentoring, Educational Services, and Learning Incentives: Evidence from a Randomized Trial in the United States. American Economic Journal: Applied Economics, v. 4, n. 4, p. 121-39, 2012.

TANG, W.; HU, T.; HU, B.; JIN, C.; WANG, G.; XIE, C. et al. Prevalence and correlates of PTSD and depressive symptoms one month after the outbreak of the COVID-19 
epidemic in a sample of home-quarantined Chinese university students. Journal of Affective Disorders, n. 274, p. 1-7, 2020.

Recebido em: 24 de novembro de 2021. Aceito em: 14 de abril de 2021. 